\title{
A Method for Measurement of the Hypertrophic First Metatarsal Medial Eminence on Foot Radiographs-A Reliability Study with Surgical Implications
}

\author{
Paul S. Armanasco ${ }^{*}$, Kathy Briffa ${ }^{2}$, Nick Pang ${ }^{3}$ \\ ${ }^{1}$ Australasian College of Podiatric Surgeons, Perth, Australia \\ ${ }^{2}$ Curtin University of Technology, Perth, Australia \\ ${ }^{3}$ Global Diagnostics, Bunbury, Australia \\ Email: ${ }^{*}$ armanascopaul@gmail.com, K.Briffa@exchange.curtin.edu.au, npang@globaldiagnostics.com.au
}

Received 9 December 2013; revised 10 January 2014; accepted 16 January 2014

Copyright $@ 2014$ by authors and Scientific Research Publishing Inc.

This work is licensed under the Creative Commons Attribution International License (CC BY). http://creativecommons.org/licenses/by/4.0/

\section{Open Access}

\section{Abstract}

Background: It has been suggested that hypertrophic eminence on the medial head of the first metatarsal is a component of the patho-anatomy of hallux valgus. However, research findings in this area are inconsistent, possibly due to differences in methods in identifying and measuring the medial eminence. To date, reliability of any method of measurement has not been reported. The purpose of this study was to determine the reliability of measurement of the medial eminence on medial oblique and dorsoplantar radiographs using a previously described geometric technique. Methods: To evaluate the reliability of a method of measurement of the medial eminence of the first metatarsal duplicate standardized dorsoplantar and medial oblique radiographs were taken of one foot of 15 subjects. The feet included both rectus feet and subjects with hallux valgus. The medial eminence was measured on the digital images using Inteleviewer 2.5 (Intelerad medical systems incorporated, Montreal, Quebec) software. Observer 1 measured one randomly selected image from each subject to determine inter-observer reliability. Observer 2 measured all images to determine the overall intraobserver reliability. Reliability was calculated using intra-class correlation coefficients (ICC). Results: The mean projection of the medial eminence ranged from 0.39 to $0.44 \mathrm{~cm}$ in both views. The ICCs were calculated for the dorsoplantar view and the 2 medial oblique view measures between observers. They ranged from 0.76 intra-rater and 0.86 interrater in the dorsoplantar view to 0.80 intra-rater and 0.90 inter-rater in the medial oblique view indicating good reliability in all measures. The average of the 4 oblique views also showed a high level of reliability with a coefficient of 0.96 . Conclusions: The medial eminence can be reliably measured in the medial oblique and dorsoplantar view using the described technique. The medial

\footnotetext{
"Corresponding author.
}

How to cite this paper: Armanasco, P.S., Briffa, K. and Pang, N. (2014) A Method for Measurement of the Hypertrophic First Metatarsal Medial Eminence on Foot Radiographs-A Reliability Study with Surgical Implications. Journal of Analytical Sciences, Methods and Instrumentation, 4, 31-37. http://dx.doi.org/10.4236/jasmi.2014.41005 
oblique and the dorsoplantar views are valuable and reliable projections for visualising the dorso medial aspect of the first metatarsal.

\section{Keywords}

\section{Hallux Valgus; Medial Eminence; Radiograph; Surgery; Reliability}

\section{Introduction}

It has been suggested that a hypertrophic eminence on the medial head of the first metatarsal is a component of the patho-anatomy of hallux valgus. However, research findings in this area are inconsistent, possibly due to differences in methods in identifying and measuring the medial eminence (ME). It appears that only two studies have examined the prevalence of this protuberance [1] [2]. Moreover, only one of these studies measured the size of the projection.

On review of the literature, the only technique that is used to quantify the ME projection was described by Thordarson and Krewer [2] (Figure 1). No other literature referring to this measurement technique and its intra-observer and inter-observer reliability were located.

In a study utilising magnetic resonance imaging including 11 people with hallux valgus, a $95 \%$ incidence of hypertrophic ME was observed [1]. However, details of the method used to measure the ME were not described and the boundary between where the eminence started and the head of the metatarsal was also not defined. As this study did not measure a control group without hallux valgus, it is difficult to interpret these findings.

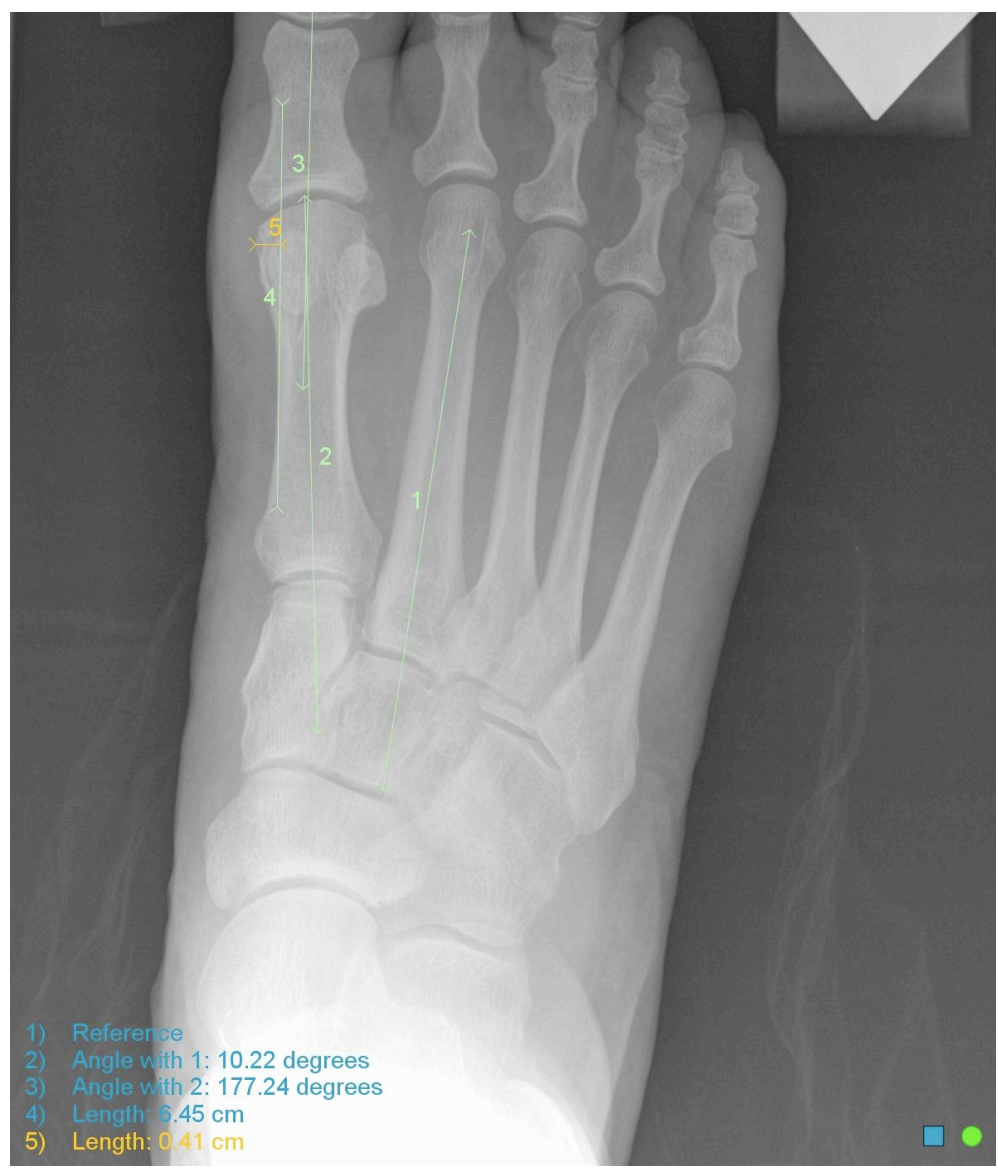

Figure 1. Method of measurement of the medial eminence projection on a weight bearing dorso plantar radiograph (line 5). 
Another study measured the projection of the ME in people with hallux valgus and hallux limitus ( $\mathrm{n}=33$ ) using weight-bearing dorsoplantar radiographic views and compared them with a control group $(n=49)$ [2]. No difference in the projection of the ME was observed and it was concluded that ME is not a component of the pathoanatomy of hallux valgus. A potential limitation of this study is that the prominence of the bunion deformity has been suggested to be located at the dorsal medial corner of the first metatarsal head, so the dorsoplantar view may not be the best radiographic method to quantify it.

Poor reliability of the method of measuring the ME will hamper the ability to detect a difference increasing the risk of Type 2 error. This is difficult to judge as reliability to date, reliability of methods of measuring the ME have not been reported. The purpose of this study was to determine the reliability of ME measurement on medial oblique and dorsoplantar radiographs. The most common pre-operative objective evaluation of hallux valgus surgery is radiological measurement [4]. The most common radiographic views are dorsoplantar and lateral views of the foot that are obtained to delineate normal and pathologic osseous and soft tissue anatomy [5]. However, as the ME projects dorsomedially it may be more evident on an oblique view [3] [6] [7]. In the oblique view, rotating the foot and the first metatarsal to a more valgus position shows both the medial dorsal components of the protuberance.

In their anatomical radiographic study of the first metatarsal, Christman and Ly [6] found that the medial eminence can be viewed tangentially with the dorsoplantar projection. The eminence is isolated and appears as a small protuberance with the lateral oblique projection (medial oblique view) and it is superimposed on the head and neck with the medial oblique and lateral projections. These authors conclude that the dorsoplantar and lateral oblique projections are the best to identify the medial eminence. However, their study was descriptive and did not report the reliability of the technique.

Consistent positioning of the foot during X-ray image acquisition is critical to minimise distortion and magnification [5], which will reduce the repeatability of radiographic measurements. It has been suggested that dorsoplantar and lateral projections can be performed more consistently than oblique projections [2]. Objective repeatability data for oblique radiographs of the foot are not available.

Geometric measurements of radiographs may be made manually or digitally. Although digital radiographs cannot improve the content of the film, the contrast and detail can be enhanced and clinical measurements can be rapidly done on the computer screen. Inter- and intra-observer reliability is comparable for manual and computer assisted measurements of hallux valgus parameters [8] [9].

By learning more about the anatomical appearance of this common anatomical feature and the optimal approach to measuring it, the basic infrastructure is set for further research in this area.

\section{Methods}

\subsection{Study Design}

A cross-sectional repeated measures study was conducted.

\subsection{Subject Recruitment}

Fifteen (11 female and 4 male) subjects were recruited from the patients attending a clinic for assessment and treatment of $\mathrm{HV}$ or other unrelated foot disorders. All subjects required radiographs for reasons related to their clinical management. The subjects were given an information sheet and a consent form outlining the nature of the study. From this information they chose to participate in the study. The samples that were included in this study were representative of the population with and without hallux valgus who required radiological assessment. The range of intermetatarsal angles was between 8 - 21 degrees and hallux valgus angle range was 7 - 56 degrees. The hallux valgus subjects included had signs and symptoms of the first metatarsophalangeal joint deformity of sufficient severity for them to seek professional consultation. The other subjects required x-rays for foot problems unrelated to hallux valgus. Subjects were excluded if they had previous history of foot surgery, trauma, inflammatory joint disease, or other syndromes, that could predispose them to hallux valgus [10].

\subsection{Procedure}

All subjects had a DP and a MO view taken. A duplicate MO was taken for 14 subjects. All subjects provided written consent to participate in the project which was approved by the Curtin University human research ethics 
committee. The dorsoplantar and lateral projections were conducted as described by Brand and Coleman's [8] standardised technique. In all views the anode to film distance was $100 \mathrm{~cm}, 15$ degrees of cephalic angulation of the tube, and the $\mathrm{x}$-ray beam centred on the midfoot. The oblique view was conducted using a rigid radiopaque wedge (Figure 2) where the foot forms an angle of 30 degrees to the plane of the film [11]. Dorsoplantar, lateral and oblique views were taken by the same experienced radiographer using the same equipment. The oblique view was taken with the subject partially weight bearing whilst sitting to ensure the foot made full contact on a radiolucent plate made of polycarbonate to prevent motion and ensure consistency. The wedge had rubber stops to prevent it slipping.

The measurement of the ME was conducted using the method described by Mann and Coughlin [3] and utilised by Thordarson and Krewer [2]. In contrast to the latter authors the ME was measured utilising digital technology on the dorsoplantar and also the medial oblique view. The radiographs were viewed and measured using inteleviewer interactive digitiser. The first metatarsal was enlarged to 150 percent to enable better visualisation however, the image was protected in its perspective and ratio. The contrast was altered if required to ensure clear delineation between soft tissue and osseous structure. The ME was quantified by finding two points and marking a line tangential to the medial shaft of the first metatarsal and another line was added parallel to this to find the most prominent aspect of the eminence. The greatest distance from this line to the medial border the ME on the radiograph was then calculated using the inteleviewer interactive digitiser to obtain geometric data. (Figures $1 \& 3$ ). Calcification within the joint capsule not attached to the metatarsal was not included in the measurement. The angular measurements were used to ensure that the linear measurement was taken at 90 degrees from the base line running parallel to the first metatarsal diaphysis.

The measurements were conducted by two independent observers, an experienced radiologist (observer 1) and an experienced podiatric surgeon (observer 2) who were familiar with the measurement technique and the software. The observers were blinded to additional clinical information and the other observer's findings. The 15 radiographs were randomised for the measurement session. Only one randomly allocated foot from each subject was used. There was no variation from the protocol at any time during the study. Observer 1 measured the dorsoplantar and one randomly selected image from each pair that was subsequently matched to the measures of the same images made by observer 2 to determine inter-observer reliability. Observer 2 measured all the randomised projections initially (dorsoplantar view and the 1st and 2ndmedial oblique radiographs) and 1 week later repeated all of the measurements to reduce the possibility of memory bias.

\section{Results}

The mean values for the medial eminence are given in Table 1 . The mean projection of the medial eminence ranged from 0.39 to $0.44 \mathrm{~cm}$ in both views. The ICCs ranged from 0.76 intra-observer (95\% confidence interval 0.44 - 0.94) and 0.86 inter-observer (95\% confidence interval 0.63 - 0.95) in the dorsoplantar view to 0.80 intra-

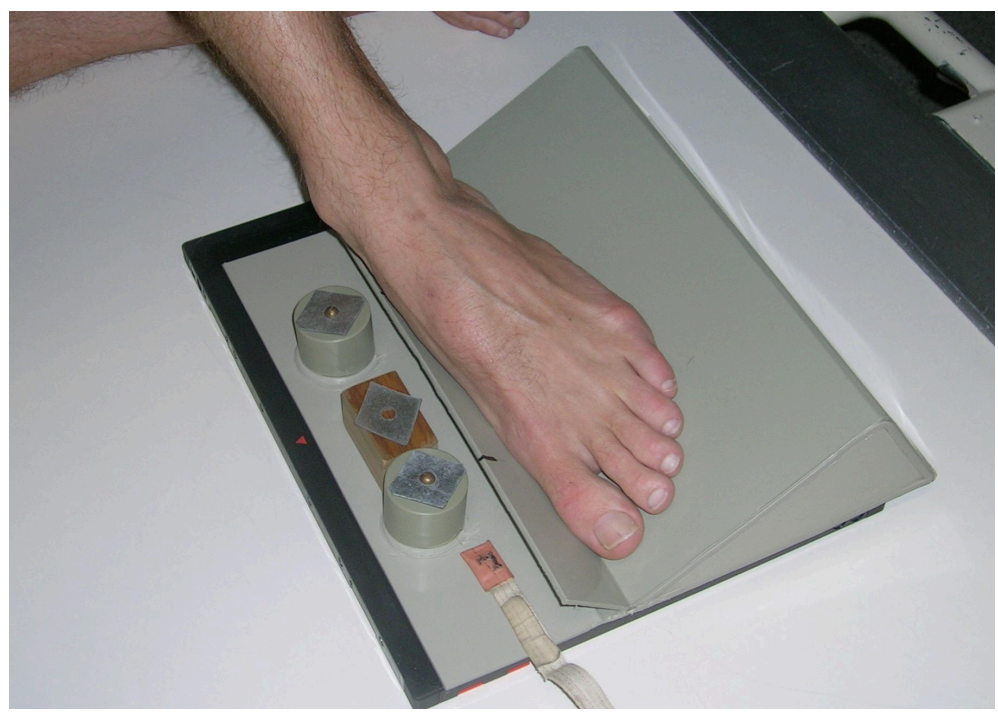

Figure 2. Method for taking the medial oblique radiograph. 


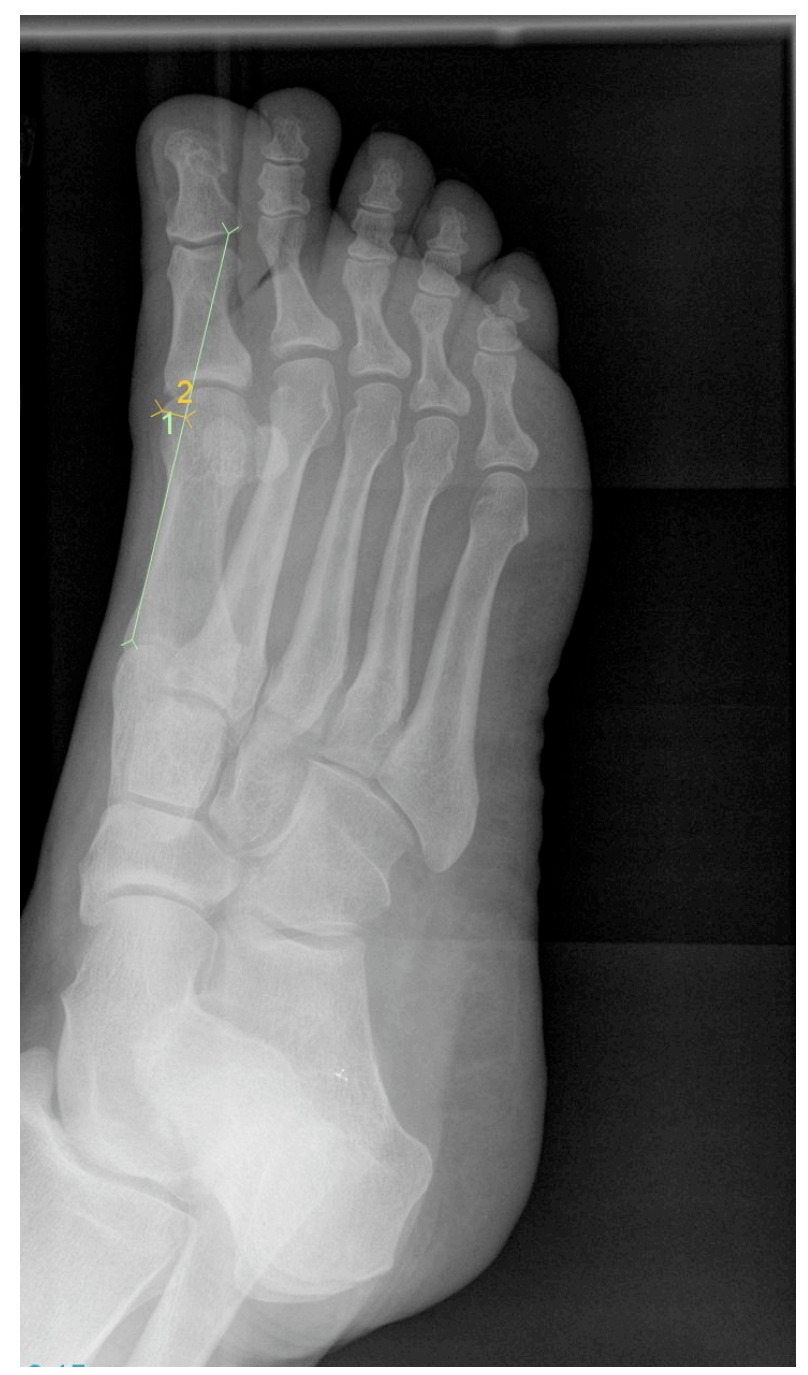

Figure 3. Method of measurement of the medial eminence projection on a medial oblique radiograph (line 2), note line 1 tangential to first metatarsal diaphysis.

Table 1. Descriptive statistics-measurement of ME with DP and MO views.

\begin{tabular}{cccccc}
\hline Projection & & & n & Mean ME(cm) & Std deviation \\
\hline DP & Observer 1 & Test 1 & 15 & 0.41 & 0.072 \\
DP & Observer 2 & Test 1 & 15 & 0.41 & 0.069 \\
DP & Observer 2 & Test 2 & 15 & 0.39 & 0.054 \\
MO & Observer 1 & Test 1 View 1 or 2 & 15 & 0.44 & 0.615 \\
MO & Observer 2 & Test 1 View 1 or 2 & 15 & 0.45 & 0.066 \\
MO & Observer 2 & Test 1 View 1 & 15 & 0.43 & 0.079 \\
MO & Observer 2 & Test 2 View 1 & 15 & 0.43 & 0.066 \\
MO & Observer 2 & Test 1 View 2 & 14 & 14 & 0.43 \\
\hline
\end{tabular}

$\mathrm{ME}=$ medial eminence, $\mathrm{DP}=$ dorsoplantar radiograph, $\mathrm{MO}=$ medial oblique. 
rater (95\% confidence interval 0.49 - 0.92) and 0.90 inter-rater (95\% confidence interval 0.73 - 0.97) in the medial oblique view indicating good reliability in all measures. The average of the 4 oblique views also showed a high level of reliability with a coefficient of 0.96 (95\% confidence interval 0.88 - 0.99). The intraobserver and interobserver ICCs, along with their upper and lower $95 \%$ confidence limits that are based on the same repeated measurements of the medial eminence are displayed in Table 2. Statistics were calculated using statistical analysis software program SPSS 13.0.

\section{Discussion}

Valid and reliable measurements provide the basis for scientific research and discussion. The ME is a deformity that has been associated with hallux valgus and is addressed in hallux surgery frequently. The ME has been defined as a bony outgrowth on the medial aspect of the head of the first metatarsal forming a bulbous medial protuberance [1]. It is routinely assessed using dorsoplantar, medial oblique and to a lesser extent on lateral radiographs.

A review of the literature revealed no data regarding the reliability of the dorsoplantar and medial oblique radiographic projections, nor regarding the measurement of the ME. No reliability studies on similar projection within the foot were located. In this publication a technique for quantification of the ME has been described using DP and MO radiographs. It has been suggested that the medial oblique is performed less consistently than dorso plantar radiographs [9] however; the results of this study would indicate that the medial oblique radiograph using the described protocol have a slightly higher reliability whilst measuring the medial eminence projection measurement in terms of both inter rater ( 0.90 compared to $0.86 \mathrm{DP})$ and intra rater repeatability (0.80 compared to $0.76 \mathrm{DP})$. These figures are considered to indicate good reliability [12]. May et al. [13] utilised a pre determined criteria for satisfactory reliability as 0.85 however they suggest that such cut off points are arbitrary. The 95\% confidence interval for the ICCs were of varying range with the largest range (0.44 - 0.91) with the dorsoplantar intra-observer reliability which does not add support to its repeatability. Interestingly the lowest $95 \%$ confidence interval $(0.88-0.99)$ was for the average of the 4 oblique views which is the most convincing in terms of reliability.

Many surgical texts describe resection of the medial head of the metatarsal with or without osteotomy in the surgical correction of the hallux valgus deformity. The question raised is whether there is a bony eminence to resect? Thordarson and Krewer [2] suggest not. Low levels of reliability may compromise the diagnostic process which could ultimately compromise clinical outcomes [13]. Knowledge of the reliability of measurement techniques is an important first step for further research to quantify the magnitude of the projection and determine whether there is a bony eminence to resect. Whilst utilising the described measurement techniques the next component of this study has the potential to add to the literature regarding this and thereby potentially influence the way that conditions involving the first metatarsal head are managed surgically.

The findings of this study need to be interpreted in the context of several limitations in its design. Firstly, al though the sample subjects were randomly allocated they were not randomly selected, so the radiographic morphology of the subjects may not be generalisable to the broader community. Secondly, the presence of systemic disease, trauma and other diseases that may predispose them to an atypical shaped first metatarsal was determined by self-report and there may have been the potential for inclusion of one of these subjects if they did not report their condition. Further research is required to repeat this study with a larger sample number and more observers to further augment the results.

Table 2. Intraclass correlation coefficients.

\begin{tabular}{ccc}
\hline Measurement & ICC & 95\% Confidence interval \\
DP Intra-rater & 0.76 & 0.44 to 0.91 \\
DP Inter-rater & 0.86 & 0.63 to 0.95 \\
MO randomised V1or 2 Intra-rater & 0.80 & 0.49 to 0.92 \\
MO randomised Inter rater & 0.90 & 0.73 to 0.97 \\
MO O2 T1 V1 \& 2 with $\mathbf{~ O 2 ~ T 2 ~ V 1 \& 2 ~}$ & 0.96 & 0.88 to 0.99 \\
\hline
\end{tabular}

$\mathrm{V}=$ view, $\mathrm{T}=$ Test, $\mathrm{O}=$ Observer. 


\section{Conclusion}

By developing a reliable tool for quantification of a morphological structure, the infrastructure is set for further research in this area utilising subjects with and without hallux valgus enabling us to learn more about this common forefoot deformity. The described technique has been shown to have good interobserver and intraobserver reliability. Ultimately, the consideration is of improvement of the quality of patient management in the clinical setting and add to the limited literature on this subject.

\section{Competing Interests}

The authors did not receive grants or outside funding in support of their research or preparation of this manuscript. They did not receive payments or other benefits or a commitment or agreement to provide such benefits from a commercial entity. No commercial entity paid or directed, or agreed to pay or direct, any benefits to any research fund, foundation, educational institution, or other chartable or non profit organisation with which the authors are affiliated or associated.

\section{Acknowledgements}

PA conducted all the measurements along with NP. KB reviewed the manuscript and assisted with research design.

\section{Author Contributions}

Roger Thornton, Peter McKinnon, Mario Horta.

\section{References}

[1] Schweitzer, M.A., Maheshwarie, S. and Shabshin, N. (1999) Hallux Valgus and Hallux Rigidus: MRI Findings. Clinical Imaging, 23, 397-402. http://dx.doi.org/10.1016/S0899-7071(00)00167-4

[2] Thordarson, D.B. and Krewer, P. (2002) Medial Eminence Thickness with and without Hallux Valgus. Foot and Ankle International, 23, 48-50.

[3] Mann, R. and Coughlin, M.J. (1994) Adult Hallux Valgus. In: Mann, R. and Coughlin, M.J., Eds., Surgery of the Foot and Ankle (6th Edition), St. Louis, Mosby, 175.

[4] Resch, S., Ryd, L., Strenstrom, A., Johnsson, K. and Kristbjorn, R. (1995) Measuring Hallux Valgus: A Comparison of Conventional Radiography and Clinical Parameters with Regard to Measurement Accuracy. Foot and Ankle International, 16, 267-270. http://dx.doi.org/10.1177/107110079501600504

[5] Bryant, J. (2001) A Comparison of Radiographic Foot Measurements Taken in Two Different Positions. Journal of the Podiatric Medical Association, 81, 234-238.

[6] Christman, R.A. and Ly, P. (1990) Radiographic Anatomy of the First Metatarsal. Journal of the Podiatric Medical Association, 90, 177-203.

[7] Martin, D. and Pontious, J. (2001) Introduction and Evaluation of Hallux Abducto Valgus. In: Banks, A.S., Downey, M.S., Martin, D.E., Miller. McGlarmry's Comprehensive Textbook of Foot and Ankle Surgery, Vol 1 (3rd Edition), Lippincott Williams and Wilkins, 484.

[8] Brand, P.W. and Coleman, W.C. (1984) A Standard for Dorsal Plantar and Lateral Radiographic Projections of the Feet. Orthopedics, 10, 117-120.

[9] Panchbhavi, V.K. and Trevino, S. (2004) Comparison between Manual and Computer-Assisted Measurements of Hallux Valgus Parameters. Foot and Ankle International, 25, 708-711.

[10] Bryant, A., Tinley, P. and Singer, K. (2000) A Comparison of Radiographic Measurements in Normal, Hallux Valgus, and Hallux Limitus Feet. The Journal of Foot and Ankle Surgery, 39, 39-43. http://dx.doi.org/10.1016/S1067-2516(00)80062-9

[11] Ballinger, P.W. (1999) Merrill’s Atlas of Radiographic Positions and Radiologic Procedures. 9th Edition, St Louis, Mosby, 177.

[12] Portney, L.G. and Watkins, M.P. (1993) Interpretation of the Intraclass Coefficients. In: Foundations of Clinical Research Applications to Practice: Appleton \& Lange, 199, 513-514.

[13] May, S., Littlewood, C. and Bishop, A. (2006) Reliability of Procedures in the Physical Examination of Non-Specific Low Back Pain: A Systematic Review. Australian Journal of Physiotherapy, 52, 91-102. http://dx.doi.org/10.1016/S0004-9514(06)70044-7 